\title{
Optimizing HER2-positive breast cancer therapy through neoadjuvant platforms: controversies and concepts
}

\author{
Shuang-Ling Wu, Ying-Ying Xu \\ Department of Breast Surgery, The First Affiliated Hospital of China Medical University, Shenyang, China \\ Contributions: (I) Conception and design: Both authors; (II) Administrative support: None; (III) Provision of study materials or patients: None; (IV) \\ Collection and assembly of data: SL Wu; (V) Data analysis and interpretation: Both authors; (VI) Manuscript writing: Both authors; (VII) Final \\ approval of manuscript: Both authors. \\ Correspondence to: Ying-Ying Xu, PhD. Department of Breast Surgery, The First Affiliated Hospital of China Medical University, No. 155, North \\ Nanjing Street, Shenyang 110001, China. Email: xuyingying@cmu.edu.cn.
}

\begin{abstract}
HER2-positive breast cancer is a highly aggressive subtype that once had a poor prognosis. While the last two decades have seen progress in multiple HER2-targeting agents that lead to improved patient outcomes, these benefits are accompanied by increased toxicity and great cost. In addition, as not all patients have the same risk of disease, individualized treatment regimens are required. Neoadjuvant therapy can allow surgical de-escalation while also providing efficacy and prognostic information, and for HER2positive breast cancer, chemotherapy combined with dual HER2-blockade regimens can achieve 42-68\% pathologic complete response (pCR) and translate to excellent outcomes. The KATHERINE study showed that adjuvant trastuzumab emtansine (T-DM1) ameliorated invasive disease-free survival (iDFS) significantly in patients with residual disease. However, the question remains as to whether treatment can be tailored for patients with pCR or early response, and several ongoing trials such as CompassHER2 and PHERGain are attempting to address this. Preoperative therapy is currently recommended by the American National Comprehensive Cancer Network (NCCN) and by Chinese Guidelines for HER2-positive breast cancer patients with $\geq \mathrm{T} 2$ or $\geq \mathrm{N} 1$. For now, we can call neoadjuvant therapy a promising platform for optimizing HER2-positive breast cancer treatment. However, it is an ongoing challenge to individualize management of anti-HER2 regimens, and this narrative review will discuss current trials and comment on several controversial topics based on therapeutic strategies and clinical practices in China.
\end{abstract}

Keywords: HER2-positive breast cancer; dual HER2-blockade therapy; adjuvant therapy

Received: 14 June 2021; Accepted: 30 June 2021; Published: 31 July 2021.

doi: $10.21037 /$ tbcr-21-10

View this article at: https://dx.doi.org/10.21037/tbcr-21-10

\section{Introduction}

Human epidermal growth factor receptor 2 overexpressing (HER2-positive, HER2+) breast cancer accounts for approximately $15 \%$ to $20 \%$ of invasive breast cancer and is an aggressive phenotype associated with high recurrence risk and poor outcome (1). The development of multiple HER2-targeting agents has progressed treatment options and improved survival, and neoadjuvant therapy, a new mode of treatment, can downgrade tumors as well as provide information on tumor response and prognosis.
Pathologic complete response (pCR) is a crucial surrogate endpoint for prognosis in HER2+ breast cancer. Patients with pCR have excellent outcomes, and while those with non-pCR have poor outcomes $(2,3)$, these have been shown to be improved with adjuvant trastuzumab emtansine (T-DM1) (4). Thus, searching for effective regimens to increase pCR and investigating appropriate strategies for residual disease are crucial for improving survival.

Currently, based on the higher pCR rates achieved in the NeoSphere (4,5) and PENOY (6) studies, chemotherapy combining trastuzumab $(\mathrm{H})$ and pertuzumab $(\mathrm{P})$ has 
become a standard neoadjuvant strategy for HER2+ breast cancer. The APHINITY trial provides additional evidence for a survival benefit with $\mathrm{H}$ and $\mathrm{P}$ in adjuvant therapy, particularly for node-positive patients $(7,8)$. In addition, the KATHERINE trial has shown that the administration of T-DMI (2) can reduce recurrence and death in patients with residual disease after neoadjuvant therapy, and several ongoing trials such as PHERGain (9) and CompassHER2 are aiming for therapeutic de-escalation by using a neoadjuvant platform.

However, many controversial questions remain, such as how can physicians balance the efficacy, toxicity, and cost of treatment while using this platform? This narrative review raises several important topics and discusses current Chinese clinical practice.

\section{Topic 1: Is it time to abandon anthracyclines from the dual HER2-blockade regimen in a neoadjuvant setting?}

Dual HER2 blockade with $\mathrm{H}$ and $\mathrm{P}$ provides superior efficacy and has become a standard regimen in neoadjuvant settings. The TRAIN-2 study (10) investigated the optimal chemotherapy backbone for dual HER2 blockade and demonstrated that addition of anthracyclines did not improve pCR for a carboplatin-taxane regimen (67\% vs. $68 \%, \mathrm{P}=0.95)(10)$, and 3 -year event-free survival (EFS) was not significantly different between the two groups (11). This raised the question of whether anthracyclines should be abandoned from dual HER2 blockade in a neoadjuvant setting and remains a highly controversial issue. While at the 2021 Consensus Conference on Early Breast Cancer Treatment Standards in St. Gallen, $53.85 \%$ of the panel voted that anthracyclines are necessary for stage II, node positive patients, three crucial factors must be considered when addressing this question. First, do anthracyclinecontaining and -free regimens provide equivalent survival? Although the TRAIN-2 and TRYPHAENA trials showed similar 3-year disease-free survival (DFS) between anthracyclines-containing and -free groups, adjuvant trials provide more sufficient survival evidence. The APHINITY trial showed that a dual HER2 blockade regimen was more beneficial for node-positive disease through 6-year follow up, in which nearly $78 \%$ patients were given anthracyclinecontaining treatment with HP. The KAITLIN (12) trial focused on high-risk HER2 + early breast cancer, and all the schemes designed contained anthracyclines. However, sample size and follow-up time for neoadjuvant studies are still insufficient for comparison with adjuvant studies. The second factor to consider is cardiac safety. The TRAIN-2 and APHINITY studies showed that anthracyclinecontaining therapy increased cardiac adverse events. The TRYPHAENA trail, which was designed to evaluate cardiac safety of dual blockade with anthracycline-containing or -free neoadjuvant regimens, demonstrated low rates of symptomatic left ventricular systolic dysfunction in both groups $(13,14)$. This confirmed that patients need to be assessed for cardiac risk and avoid anthracyclines in highrisk conditions. The third factor arises when considering that HER2+ breast cancer is a heterogenous disease: could strengthening anti-HER2 therapy have varied effectivity depending on the intrinsic subtype? For example, as a subset of triple-positive breast cancer tumors have biological behavior more like luminal phenotypes, they may be less sensitive to anti-HER2 therapy.

Therefore, it is too early to say whether anthracyclines should be totally abandoned from dual HER2-blockade in neoadjuvant setting, and further investigation is required, especially in high-risk or ER+/HER2 + breast cancer patients.

\section{Topic 2: What is the optimal adjuvant regimen for HER2-positive breast cancer after pCR based on dual HER2-blockade?}

Experts from China and other countries hold differing views regarding this question. Recognizing the pCR achieved by neoadjuvant chemotherapy with $\mathrm{HP}$ in $\mathrm{cN}+$ patients, 55.56\% of the St. Gallen 2021 panel voted for HP to be used in adjuvant regimens. However, in $\mathrm{cN} 0$ patients, $69.64 \%$ of the panel preferred $\mathrm{H}$ for adjuvant settings. This recommendation may arise from the APHINITY study, in which the addition of pertuzumab to chemotherapy with trastuzumab improved invasive disease-free survival (iDFS) significantly in the node-positive cohort (87.9\% vs. 83.4\%), but not in the node-negative cohort (95\% vs. $94.9 \%)$ after a 6-year follow up (8).

Despite this, a crucial factor may be missing from these analyses. Lymph node status was confirmed by pathology after surgery in APHINITY, providing a true positive or negative status. However, while patients are undergoing neoadjuvant treatment, baseline lymph nodes are evaluated clinically, meaning that only suspected nodes will be further verified by ultrasound-guided biopsy. Discrepancies between clinical and pathological diagnosis have been reported, with the sensitivity and specificity 
of positive axillary lymph node detection with ultrasound only $49-87 \%$ and $55-97 \%$, respectively (15). Moreover, clinical practice differs between China and Western countries. Patients with larger tumors and $\mathrm{cN} 0$ will often receive neoadjuvant therapy in China, leading to a higher chance of underestimating positive lymph node status. A meta-analysis in SABCS 2019, including the HannaH, NeoSphere, TRYPHENA, BERENICE, and KRISTINE studies, showed that the 3-year EFS of pCR patients in $\mathrm{HP}$ (neoadjuvant) to $\mathrm{H}$ (adjuvant) was $89.7 \%$, and $\mathrm{HP}$ (neoadjuvant) to HP (adjuvant) was $96.9 \%$ (16). On this basis, Chinese experts are more likely to agree with HP as an adjuvant regimen after pCR based on dual HER2blockade. The Guidelines of the Chinese Society of Clinical Oncology (CSCO) also support continuation of the original effective neoadjuvant targeted drugs in an adjuvant setting.

\section{Topic 3: How to develop adjuvant strategies for HER2-positive breast cancer after non-pCR based on dual HER2-blockade}

Patients with residual disease after dual HER2-blockade account for $30-60 \%$ of cases and have poor outcomes (17). The KATHERINE study demonstrated the 3-year iDFS of non-PCR patients was significantly higher following adjuvant T-DM1 than trastuzumab $(88.3 \%$ vs. $77.0 \%$, $\mathrm{P}<0.001)$. T-DM1 has been recommended by international guidelines for HER2+ breast cancer patients who have residual disease after standard neoadjuvant therapy.

Should patients with an excellent clinical response, for instance less than $5 \mathrm{~mm}$ of residual invasive cancer, be offered adjuvant T-DM1? In 2021, 77.36\% of the panel of St. Gallen voted in favor, but Chinese experts hold different opinions. In the KATHERINE trial, only $17.9 \%$ of all patients received dual HER2-targeted therapy with HP, a very limited number compared to the trastuzumab group. Further, positive lymph node patients achieved a 3-year iDFS of $92 \%$ from $\mathrm{H}$ and $\mathrm{P}$ based adjuvant regimen in the APHINITY trial. Unfortunately, there is no current headto-head study on HP and T-DM1 in patients with residual disease, especially for those who have achieved excellent effects from neoadjuvant therapy. Regarding safety, more grade 3 or higher platelet count decreases and hypertension occurred with T-DM1 in the KATHERINE trial, and previous studies have proposed that the incidence of grade 3-4 platelet count decreases from T-DM1 was higher in Asian patients than non-Asian patients (18). The adverseevent profile was generally balanced between HP and trastuzumab alone in APHINITY. In addition, pertuzumab has been approved under Chinese basic medical insurance, but the cost of T-DM1 limits its application in clinics, especially in developing countries.

Therefore, adjuvant strategies for residual disease should be further evaluated. Based on several conditions, the CSCO breast cancer guidelines recommend HP for patients whose tumors regress significantly (Miller \& Payne 3-4), and T-DM1 for those which do not regress significantly (Miller \& Payne 1-2).

\section{Conclusions}

Opportunities and challenges co-exist for HER2-positive breast cancer in the post-trastuzumab era. Neoadjuvant therapy is a good platform for treatment optimization. While the TRAIN-2 study analyzed concepts of deescalation of anthracyclines under dual HER2- blockade, it is difficult to say whether nine cycles of TCbHP is less toxic than standard regimens. The real de-escalation will be based on the sensitivity to anti-HER2 therapy, which may be predicted by tumor intrinsic subtype, intra-tumor heterogeneity, tumor-infiltrating-lymphocytes (TILs), and other biomarkers. T-DM1 reduced the $50 \%$ recurrence risk of invasive breast cancer among patients with residual disease, and those with high HER2 mRNA expression post-surgery saw more benefit from adjuvant T-DM1. However, its high cost and low accessibility limit its use in low and middle-income regions. HP schemes are frequently recommended by Chinese clinicians when tumors shrink significantly. Residual disease re-evaluation and guided treatment will hopefully become reasonable standards in the future.

\section{Acknowledgments}

We acknowledgment Barry Draper for the language editing of this article.

Funding: None.

\section{Footnote}

Conflicts of Interest: Both authors have completed the ICMJE uniform disclosure form (available at https:// dx.doi.org/10.21037/tbcr-21-10). YYX serves as the unpaid editorial board member of Translational Breast Cancer Research from May 2021 to April 2023. The other author has no conflicts of interest to declare. 
Ethical Statement: The authors are accountable for all aspects of the work in ensuring that questions related to the accuracy or integrity of any part of the work are appropriately investigated and resolved.

Open Access Statement: This is an Open Access article distributed in accordance with the Creative Commons Attribution-NonCommercial-NoDerivs 4.0 International License (CC BY-NC-ND 4.0), which permits the noncommercial replication and distribution of the article with the strict proviso that no changes or edits are made and the original work is properly cited (including links to both the formal publication through the relevant DOI and the license). See: https://creativecommons.org/licenses/by-nc-nd/4.0/.

\section{References}

1. Krishnamurti U, Silverman JF. HER2 in breast cancer: a review and update. Adv Anat Pathol 2014;21:100-7.

2. von Minckwitz G, Huang CS, Mano MS, et al. Trastuzumab Emtansine for Residual Invasive HER2Positive Breast Cancer. N Engl J Med 2019;380:617-28.

3. Cortazar P, Zhang L, Untch M, et al. Pathological complete response and long-term clinical benefit in breast cancer: the CTNeoBC pooled analysis. Lancet 2014;384:164-72.

4. Gianni L, Pienkowski T, Im YH, et al. Efficacy and safety of neoadjuvant pertuzumab and trastuzumab in women with locally advanced, inflammatory, or early HER2-positive breast cancer (NeoSphere): a randomised multicentre, open-label, phase 2 trial. Lancet Oncol 2012;13:25-32.

5. Gianni L, Pienkowski T, Im YH, et al. 5-year analysis of neoadjuvant pertuzumab and trastuzumab in patients with locally advanced, inflammatory, or early-stage HER2-positive breast cancer (NeoSphere): a multicentre, open-label, phase 2 randomised trial. Lancet Oncol 2016;17:791-800.

6. Shao Z, Pang D, Yang H, et al. Efficacy, Safety, and Tolerability of Pertuzumab, Trastuzumab, and Docetaxel for Patients With Early or Locally Advanced ERBB2Positive Breast Cancer in Asia: The PEONY Phase 3 Randomized Clinical Trial. JAMA Oncol 2020;6:e193692.

7. von Minckwitz G, Procter M, de Azambuja E, et al. Adjuvant Pertuzumab and Trastuzumab in Early HER2Positive Breast Cancer. N Engl J Med 2017;377:122-31.

8. Piccart $M$, Procter $M$, Fumagalli D, et al. Adjuvant Pertuzumab and Trastuzumab in Early HER2-Positive
Breast Cancer in the APHINITY Trial: 6 Years' FollowUp. J Clin Oncol 2021;39:1448-57.

9. Pérez-García JM, Gebhart G, Ruiz Borrego M, et al. Chemotherapy de-escalation using an 18F-FDGPET-based pathological response-adapted strategy in patients with HER2-positive early breast cancer (PHERGain): a multicentre, randomised, openlabel, non-comparative, phase 2 trial. Lancet Oncol 2021;22:858-71.

10. van Ramshorst MS, van der Voort A, van Werkhoven ED, et al. Neoadjuvant chemotherapy with or without anthracyclines in the presence of dual HER2 blockade for HER2-positive breast cancer (TRAIN-2): a multicentre, open-label, randomised, phase 3 trial. Lancet Oncol 2018;19:1630-40.

11. van der Voort A, van Ramshorst MS, van Werkhoven ED, et al. Three-Year Follow-up of Neoadjuvant Chemotherapy With or Without Anthracyclines in the Presence of Dual ERBB2 Blockade in Patients With ERBB2-Positive Breast Cancer: A Secondary Analysis of the TRAIN-2 Randomized, Phase 3 Trial. JAMA Oncol 2021. [Epub ahead of print]. doi: 10.1001/ jamaoncol.2021.1371.

12. Harbeck N, Im SA, Barrios CH, et al. Primary analysis of KAITLIN: A phase III study of trastuzumab emtansine (TDM1) + pertuzumab versus trastuzumab + pertuzumab + taxane, after anthracyclines as adjuvant therapy for highrisk HER2-positive early breast cancer (EBC). J Clin Oncol 2020;38:500.

13. Schneeweiss A, Chia S, Hickish T, et al. Long-term efficacy analysis of the randomised, phase II TRYPHAENA cardiac safety study: Evaluating pertuzumab and trastuzumab plus standard neoadjuvant anthracycline-containing and anthracycline-free chemotherapy regimens in patients with HER2-positive early breast cancer. Eur J Cancer 2018;89:27-35.

14. Schneeweiss A, Chia S, Hickish T, et al. Pertuzumab plus trastuzumab in combination with standard neoadjuvant anthracycline-containing and anthracycline-free chemotherapy regimens in patients with HER2-positive early breast cancer: a randomized phase II cardiac safety study (TRYPHAENA). Ann Oncol 2013;24:2278-84.

15. Alvarez S, Añorbe E, Alcorta P, et al. Role of sonography in the diagnosis of axillary lymph node metastases in breast cancer: a systematic review. AJR Am J Roentgenol 2006; 186:1342-8.

16. Swain S, Macharia H, CortÈs J, et al. Abstract P1-18-01: Risk of recurrence and death in patients with early HER2- 
positive breast cancer who achieve a pathological complete response (pCR) after different types of HER2-targeted therapy: A retrospective exploratory analysis. Cancer Res 2020;80:(4 Suppl).

17. Takada M, Toi M. Neoadjuvant treatment for HER2-

doi: $10.21037 /$ tbcr-21-10

Cite this article as: $\mathrm{Wu} \mathrm{SL}, \mathrm{Xu}$ YY. Optimizing HER2positive breast cancer therapy through neoadjuvant platforms: controversies and concepts. Transl Breast Cancer Res 2021;2:19. positive breast cancer. Chin Clin Oncol 2020;9:32.

18. Diéras V, Harbeck N, Budd GT, et al. Trastuzumab emtansine in human epidermal growth factor receptor 2-positive metastatic breast cancer: an integrated safety analysis. J Clin Oncol 2014;32:2750-7. 